\title{
Two Dimensional Electronic Spectroscopy
}

\author{
Graham R. Fleming, ${ }^{,}$Mino Yang, ${ }^{\ddagger}$ Ritesh Agarwal, ${ }^{\ddagger}$ Bradley S. Prall, Laura J. Kaufman, ${ }^{\ddagger}$ and Fred Neuwahl ${ }^{\$}$
}

\author{
Department of Chemistry, Lhiversity of California. Berkelev and the Phwsical Biosciences Division, \\ Larrence Berkelev Vational Laboratorv. Berkelev. CA 94720. LSA \\ Received.April 14, 2003
}

\begin{abstract}
Two different electronically resonant two-dimensional spectroscopies are described. The first. two-color photon echo peak shift spectroscopy. is sensitive to correlations in transition frequency between the initial and probed (final) states. It provides new insight into the mechanism of ultrafast solvation and should prove useful for characterizing dynamics in inhomogeneous șistems in general. The second teclunique, fifth order threepulse scattering. contains two coherence periods whose durations are controlled. The entire two-dimensional surface was recorded for a dye molecule in dilute solution and a photoșththetic light-harvesting complex. The data provide insight into the short-time dỵnamics of solvation and exciton relaxation. respectively:
\end{abstract}

Key Words : Non degenerate, Photon echo, Two-color, Fifth-order

\section{Introduction}

The development of stable and reliable sources of femtosecond light pulses tunable throughout the visible and infrared spectral regions has triggered a remarkable burst of activity in the development of ultrafast nonlinear optical spectroscopies. In this article, we will focus on electronically resonant spectroscopies. First to be developed were two-and three-pulse photon echo electronic spectroscopies for the study of spectral broadening and solvation dynamics in solution. ${ }^{1.8}$ The three-pulse photon echo peak shift or 3PEPS method was shown to be a simple and reliable technique for the study of the transition frequency correlation function, ${ }^{9-11}$ and was soon applied to energy transfer in both weakly coupled $^{12.15}$ and strongly coupled ${ }^{16.18}$ molecular aggregates. in particular photosynthetic light harvesting conplexes. An attractive feature of photon echo techniques in general, and 3PEPS in particular, is the ability to undo the ensemble average over transition frequencies that gives rise to spectral broadening in condensed phases. Indeed the inhomogeneous width can be determined ${ }^{10}$ and exploited to study energy migration. ${ }^{1030}$ However. there are a number of aspects of condensed phase dýnamics that are not accessible via simple photon echo spectroscopies. In this paper. we surver a number of these aspects and propose that two-dimensional electronic correlation spectroscopies ${ }^{21.24}$ are a useful route to address these issues. We then describe two different types of 2-D electronic spectroscopy: fifth order three pulse photon echo spectroscopy (F3PE or FOTS ${ }^{2 \geq 5}$ ) and two-color threepulse photon echo spectroscopy (2C3PEPS ${ }^{20}$ ). In the

\footnotetext{
${ }^{\dagger}$ Present Address: Dept. of Chemistry and Institute of Basic Sciences Research, Chungbuk National University, Cheonglu, Chungbul- 361-763, Korea

tPresent Addresses: Dept. of Chemistry and Chemical Biology, Harvard University, Cambridge, MA 02138 , USA

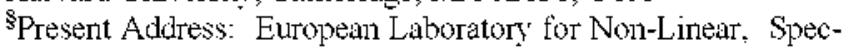
troscopy (LENS), University of Florence, Polo Scientifico, Via Nello Carara 1, 1-50019-Sesto-Fiorentino, FIRENZE Italy
}

former technique, the duration of two coherence periods is directly controlled, in the latter. one coherence period is scanned at fixed values of a population period and twodimensional information is obtained via control over the center frequency of the final light field, with respect to the first two light fields. ${ }^{26}$

Linear, Nonlinear and Multidimensional Spectroscopies Linear absorption spectra of condensed phase systems are often thought of in terms of inhomogeneous (very slow dynamics) and homogeneous (very rapid dynamics) contributions. From a linear spectrum it is impossible to uniquely separate these contributions. Nonlinear spectroscopy, in particular photon echo spectroscopy. makes it possible to remove the slow contributions to line broadening. Rather than describing the homogeneous dynamics as infunitely fast. a more physically realistic description is in terms of a 'spectrum' of hanmonic oscillators coupled to the electronic excitation. ${ }^{2728}$ By coupled we simply mean that the equilibriun coordinate value is different when the system is in its ground or excited electronic state. The spectral density is then defined by

$$
\rho(\omega)=\frac{1}{2 \pi} \sum_{i} \frac{c_{i}^{2}}{M_{i} \omega_{i}^{3}} \delta\left(\omega-\omega_{0}\right)
$$

with $c_{2}$ the coupling strength of mode $i$ to the observable of interest (e.g. the electronic energy gap). and $M_{i}$ and $\omega_{h}$ the mass and frequency of mode $i$.

A spectral density of the form given in (1) can be obtained from computer simulation, theory or empirical models. It can then be used to calculate the lineshape function, $g(t)$.

$$
\begin{aligned}
g(t)= & -i \lambda t / \hbar+\int_{0}^{\infty} d \omega \rho(\omega) \operatorname{coth}[\hbar \omega \beta / 2](1-\cos \omega t) \\
& +i \int_{0}^{\infty} d \omega \rho(\omega) \sin \omega t
\end{aligned}
$$

The nonlinear response function $R\left(t_{3}, t_{2}, t_{1}\right) i^{28}$ can then be 
calculated from $g(t)$. to give the nonlinear optical signal.

The dynamical content of $\rho(\omega)$ can be appreciated by considering the fluctuating transition frequency. $\omega_{e g}^{s}(t)$. of a single chromophore. $i$ :

$$
\omega_{e g}^{s}(t)=\left\langle\omega_{e g}\right\rangle+\delta \omega_{\varepsilon g}^{\prime}(t)+\varepsilon
$$

here $\left\langle\omega_{e g}\right\rangle$ is the average transition frequency of molecules of type $i . \varepsilon^{i}$ is an offset from this mean (i.e. the distribution of $\varepsilon$ values reflects the inhomogeneous distribution), and $\delta \omega_{e g}^{L}(t)$ describes the time-fluctuating component of the transition frequency of molecule $i$. The dynamical behavior in the fluctuations are described by a time correlation function

$$
\frac{\left\langle\delta \omega_{g g}(t) \delta \omega_{e g}(0)\right\rangle}{\left\langle\delta \omega_{g g}^{2}\right\rangle}=S(t)
$$

Here the angle brackets denote an ensemble average and the fluctuations are considered identical for all molecules, $i$. For simplicity, we focus on the classical form of equation (4). ${ }^{29}$ In ternts of the spectral density $S(t)$ can be rewritten as

$$
S(t)=\frac{\hbar}{\lambda} \int_{i}^{\infty} d \omega \omega \rho(\omega) \cos \omega t
$$

Use of equations (4) or (5) to describe the behavior of a complex system is equivalent to assuming that the spectral density is homogeneous in various senses. Indeed in conventional (one-dimensional) nonlinear optical experiments the calculation of the nonlinear response function $R\left(t_{3}+t_{2} t_{1}\right)$ via

$$
\rho(\omega) \rightarrow g(t) \rightarrow R\left(t_{3}, t_{2}, t_{1}\right)
$$

cannot provide information on whether $R\left(t_{3}, t_{2} t_{1}\right)$ is spatially homogeneous or not. In other words. $R\left(t_{3}, t_{2}, t_{1}\right)$ may be of the form

$$
R\left(t_{3}+t_{2}+t_{1}, \Gamma\right)=\int d \Gamma W(\Gamma) R\left(t_{3}, t_{2}, t_{1}\right)
$$

where $\Gamma$ is a set of parameters on which $R$ depends. but the nonlinear experiment is insensitive to the form (or even the existence) of $W(\Gamma)$. In this sense. the conventional photon echo spectroscopies give (generally wa iterative fitting procedures) a spectral density, $\rho(\omega)$. for a particular experiment that has the same relationship to the nonlinear experiment that the ordinary absorption spectrum does to linear spectroscopy.

Two specific examples may help to make this point clear. They exemplify two different types of spatial heterogeneity in the spectral density. In the first example. we consider the fluctuations in the electric potential felt by a polar molecule buried in the interior of a protein molecule which. in tum. is solvated by water. Specifically, we consider 3PEPS data for the dy'e eosin. bound to the hydrophobic box of lysozyme in comparison to similar data for the dye in aqueous solution. Figure 1 shows the structure of the complex and the 3PEPS data for the complex and the dye in solution. Clearly. (a)
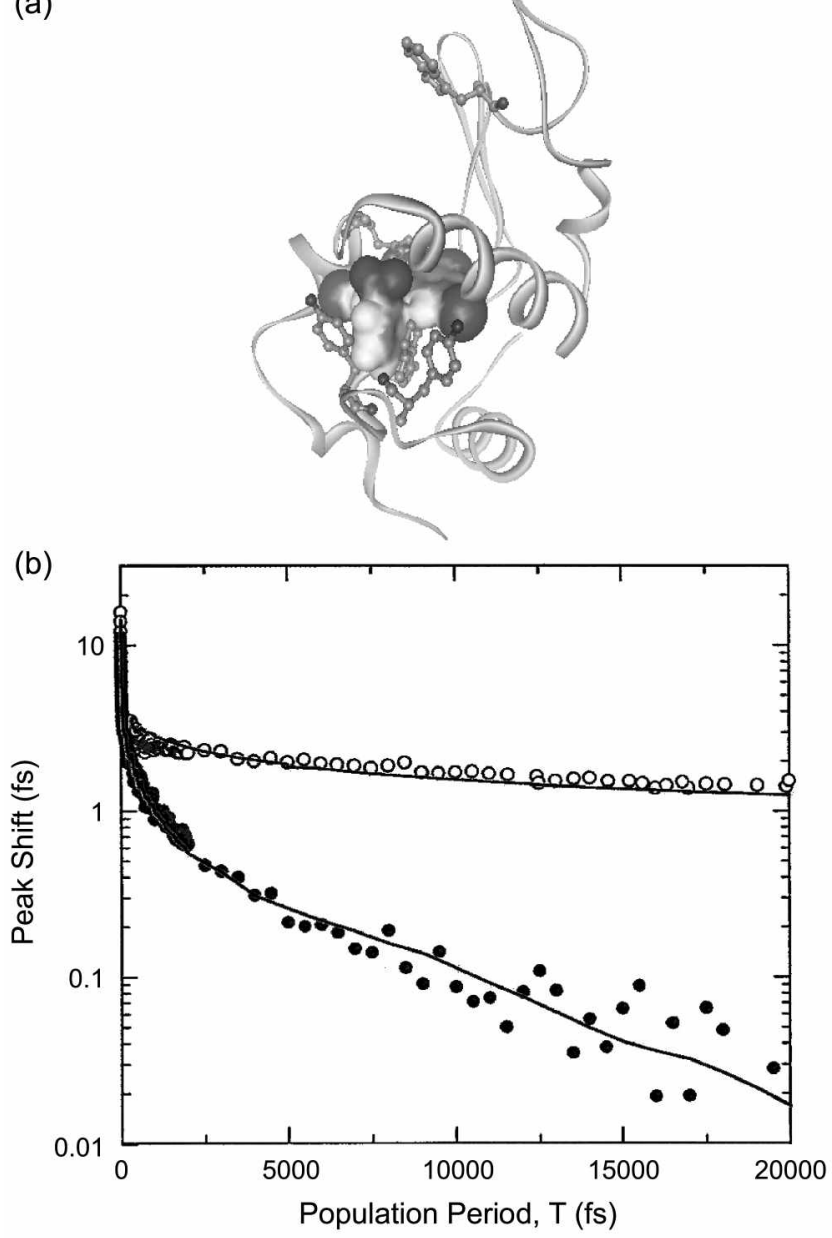

Figure 1. (a) Proposed structure of lyzozyme-eosin complex. Eosin is believed to bind within the "hydrophobic pocket" defined by residues Trp 28, Trp 111, Tyr 22, and Met 105. (b) 3PEPS data for lyzozyme-eosin complex in water (open circles) and eosin in water (solid circles ). Fits for both data sets shown as solid lines.

the peak shift decays much more slowly for the complex. although the ultrashort timescale is very similar in both systems. Detailed analysis shows that the wide range of timescales are present in the decay of the transition frequency correlation function of the dye/protein system. The solid lines in Figure lb are calculated from dielectric models of water ${ }^{31}$ or the aqueous protein complex. ${ }^{30}$ Clearly. they both describe the data quite well. yet they are constructed in quite contrasting manners in tenms of the spectral density described above. In the case of the aqueous solution. an homogeneous frequency dependent dielectric response. $\varepsilon(\omega)$. was constructed from infrared and dielectric response data. $\varepsilon(\omega)$ is then used to calculate $\rho(\omega)$ and the assumption leading from equation (3) to equation (4) or embodied in equation (6) seem well justified.

In contrast. the spectral density constructed for the protein data is quite spatially heterogeneous as shown in Figure 2. Figure 2a depicts a hierarchy of models in which the bulk water, the bound water, and the protein itself are all assigned distinct forms of $\varepsilon(\omega)$. The associated spectral densities are 
(a)
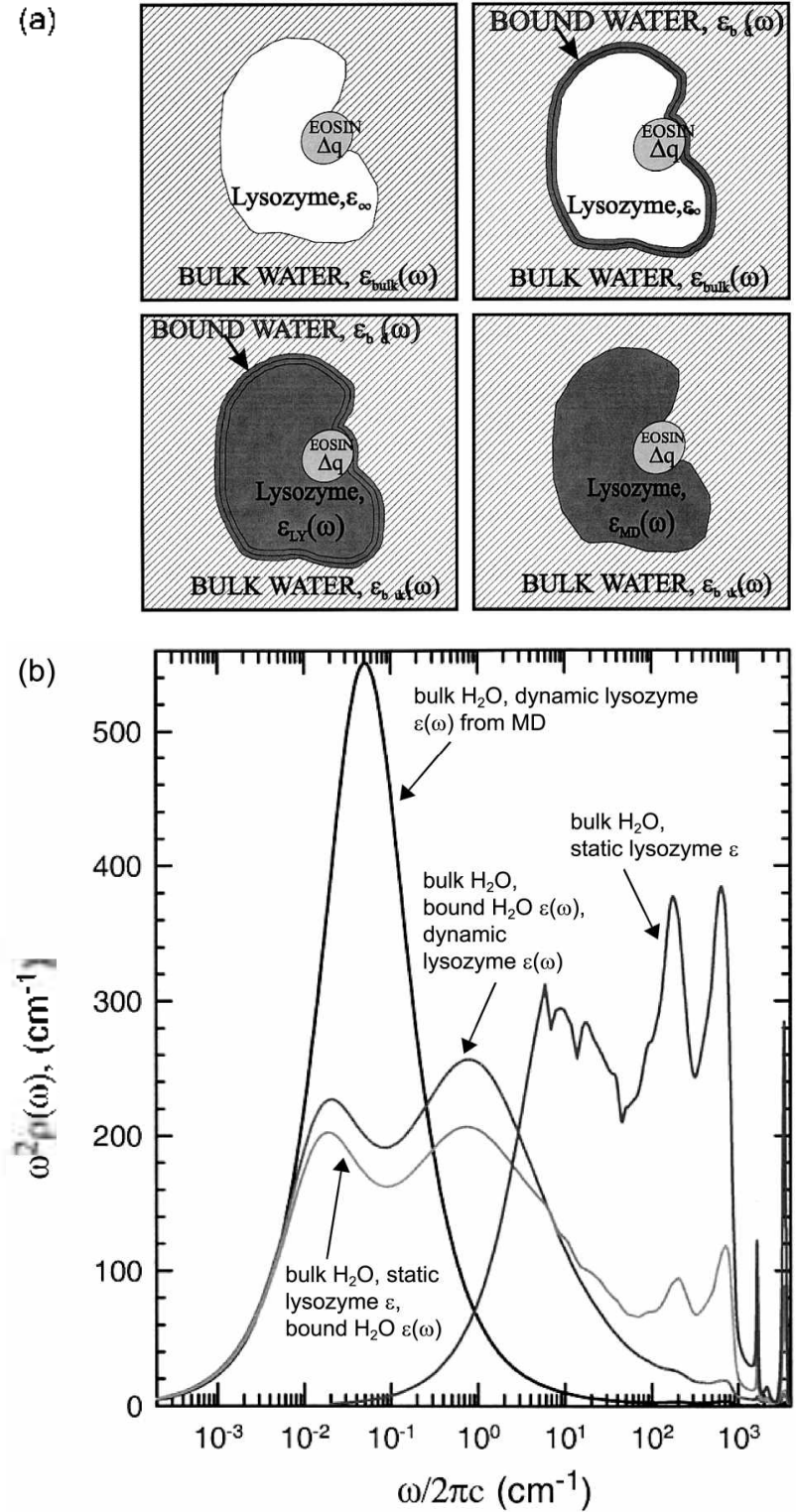

Figure 2. (a) Schematic representation of the dielectric models used for simulating the peak shift data of aqueous lyzozyme-eosin complex. Models range from treating the system as a static dielectric cavity in bulk water to including the dielectric response of the Lvzozyme, 2 lavers of bound water, and the bulk water. (b) Spectral densities for aqueous lyzozyme-eosin complex derived from the four models.

shown in Figure 2b and compared with $\rho(\omega)$ obtained from a computer simulation of the same system. ${ }^{30}$ which does not greatly resemble the model spectral densities. The solid curve in Figure lb comes from the model including bulk water. bound water. and a dielectric response for the lysozy'me itself ${ }^{30}$ All three responses are manifest in the 3PEPS decay. However, success in fitting a single peak shift decay. no matter how complex in form. with such a complicated model is clearly not an adequate test of the model's validity. Progress could perhaps be made by two dimensional experiments of the type proposed by Cho and coworkers. ${ }^{j 2}$ for example optical/infrared or optical/Raman 2D experiments.
Such studies can. in principle. determine the spectral density of those solvent molecules directly coupled to optical excitation of the chromophore. ${ }^{33}$ A related experiment has recently been described by $\mathrm{D}$. Blank and given the name RAPTORS. ${ }^{34}$

A second, and much more complex. example embodies many of the difficulties that a fully fledged electronic correlation spectroscopy might hope to address. This example is Photosystem I (PSI) of green plant and cyanobacterial photosynthetic systems (Fig. 3). ${ }^{35}$ The function of PSI is to absorb solar photons and transfer the excitation to a primary electron donor ( $\mathrm{P} 700$ ) with exquisite efficiency. to initiate the chemical steps of photosynthesis. ${ }^{36}$ Much of our detailed understanding of energy flow in photosynthesis is based on the structure of the purple bacterial light harvesting complex LH2. ${ }^{37.41}$ This is a highly symmetrical structure with just two 'types' of bacteriochlorophyll molecules in a repeating eight or nine-fold synmetric arrangement. ${ }^{37}$ In contrast. PSI contains 96 non-equivalent chlorophyll a (Chla) molecules with a wide range of site energies. inter-Chl spacings and orientations. Figure 4 shows the spectrum of a single molecule of Chla compared to the PSI complex spectrum. The PSI spectrum is very broad. highly congested and inhomogeneous. In addition. the chromophores are excitonically coupled to a greater or lesser extent. The stronger the electronic coupling the greater the charge transfer character of the resulting excitonic states is likely to be. This will lead to some correlation of the spectral density associated with the optical excitation and the wavelength of the transition. Hole buming spectroscopy has provided some direct evidence for this assertion. ${ }^{41}$ Again the assumption leading from equ. (3) to Eq. (4) is violated by this effect.

A second issue is the "chicken and egg' question of site energies and electronic couplings. In conventional spectroscopies if the site energy is not known it is not possible to unambiguously obtain the electronic coupling from. for example, the circular or linear dichroism spectra. Electronic

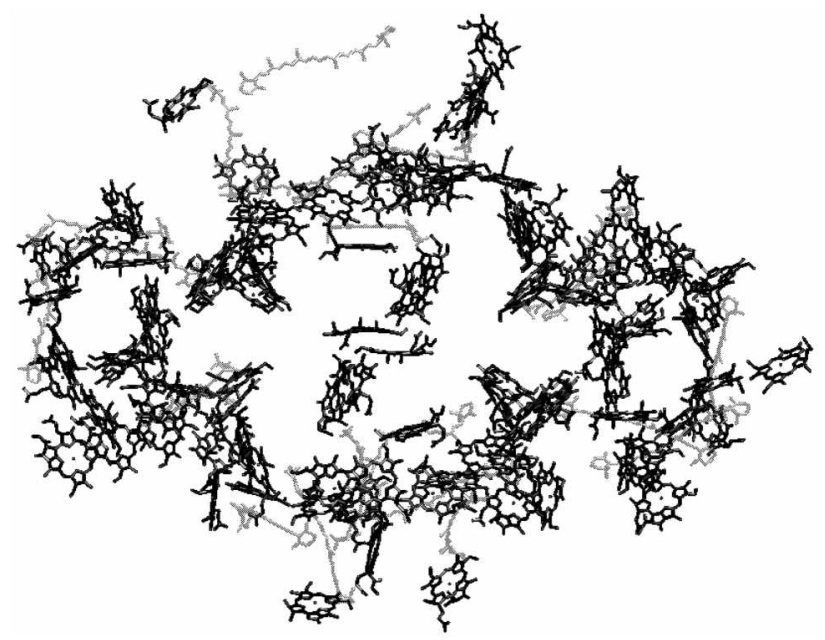

Figure 3. Positions of 96 chlorophyll a molecules (dark) as $w$ ell as carotenoids (light) bound in Photosystem I (PSI) as detemined from the crystal structure of Jordan etal. in reference 35. 


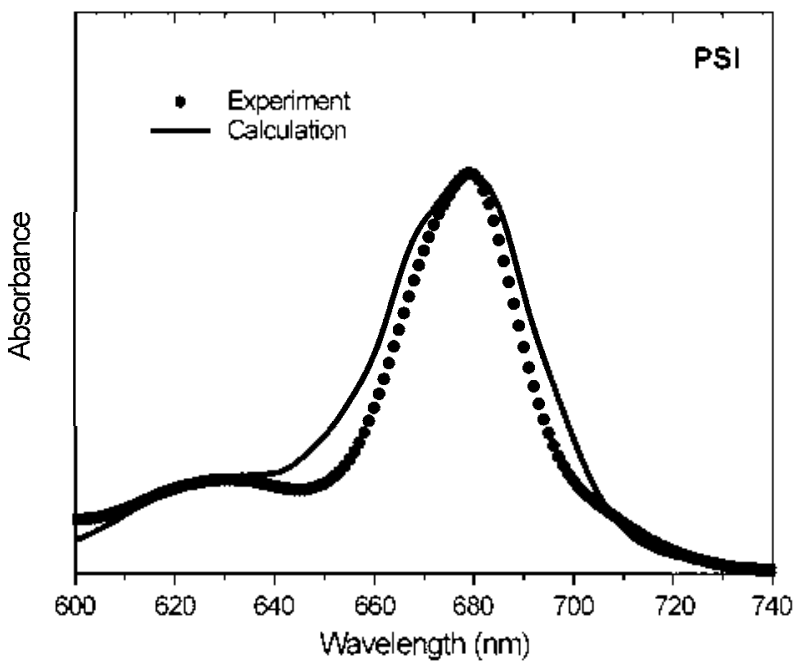

acceptor states in a disordered energy transfer system such as PSI. Later in this paper. we will describe experimental and theoretical results for the former system and here we sketch the ideas relevant to the energy' transfer case.

In considering the nonlinear optical response of a disordered energy' transfer system. the overall signal arises from a product of the energy-dependent phase factor. $\phi(\Gamma)$. and the energy-dependent population kinetics. $S(\Gamma)$. The overall response for the ensemble is written

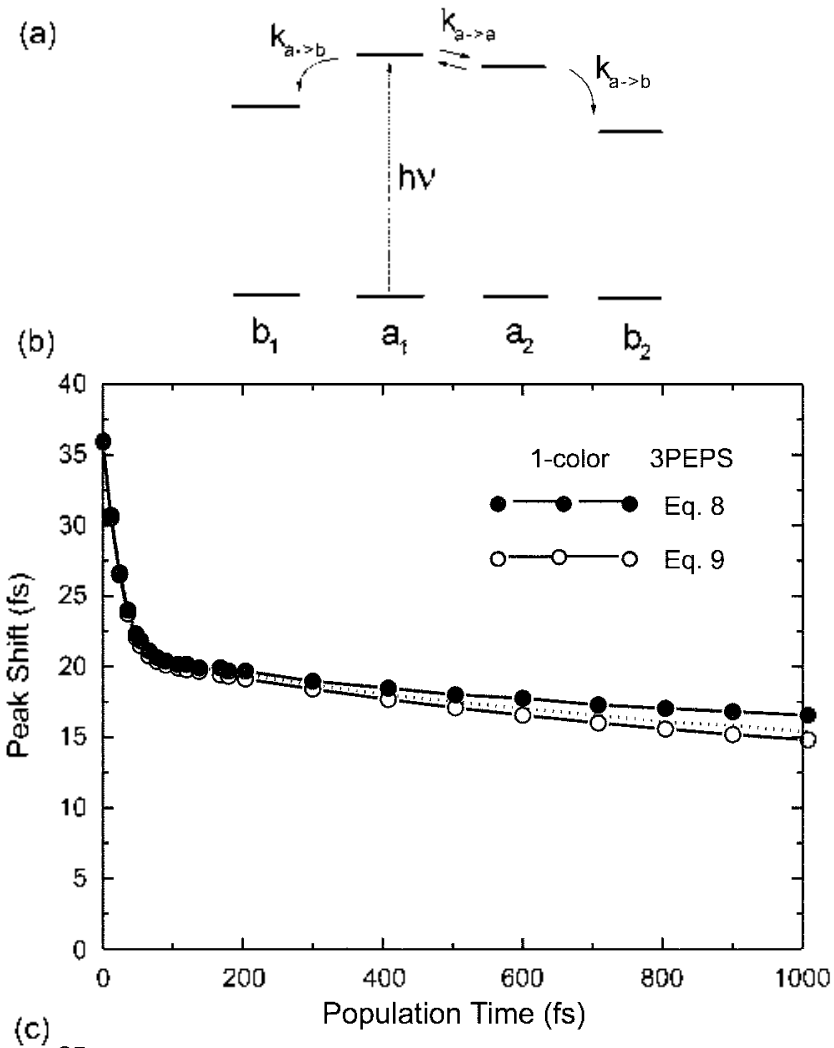

(a) Experimental room temperature PSI absorption spectra in chlorophyll a region (solid circles) along with fit from theoretical calculations of 96 chlorophyll's transition energies. (b) Calculated absorption spectra for chlorophyll a using model spectral density.

correlation spectra of the type proposed by Mukamel. ${ }^{+2}$ or the experimentally somewhat simpler two-color three-pulse photon echo spectroscopy proposed by Yang and Fleming 55 do have the potential to determine the extent of electronic mixing in molecular complexes. For example. Yang and Fleming studied a dimer system with two distinct absorption bands corresponding to the two mixed states of the dimer. They showed that by making suitable combinations of oneand two-color peak shift measurements it is possible to determine the mixing coefficient. independent of knowledge of the original site energies or the electron-phonon coupling strength and consequent exciton relaxation dy'namics. ${ }^{\text {s }}$

An unexpected aspect of two-color three-pulse echoes (in particular the 2-color 3PEPS 2PEPS (2C3PEPS) experiment proposed by Yang and Fleming). is its ability to detect correlation between the initial and final states of a dy'namical system. These two states may correspond. for example to the initial 'Franck-Condon' and solvent equilibrated excited state of a polar solute in a polar solvent. or to donor and

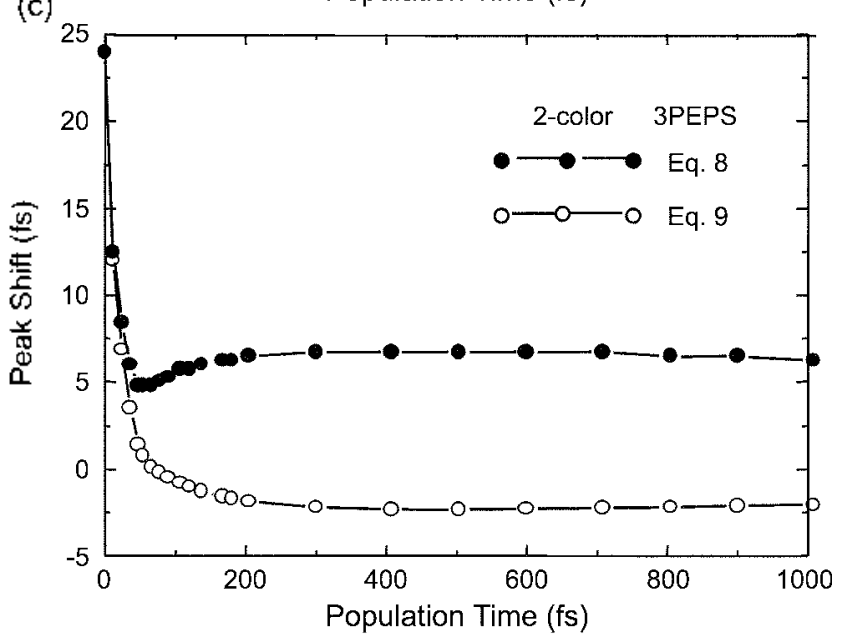

Figure 5. (a) Model system for studying energy transfer in disordered systems effect on nonlinear signals. Model contains 4 individual molecules 2 of type a and 2 of type b. The type a molecules are coupled to each other and each to a different type $b$ molecule. (b) Single Color 3PEPS calculated from equation 8 (solid circles) and equation 9 (open circles). (c) Two color 3PEPS calculated from equation 8 (solid circles) and equation s(open circlesj. 


$$
S_{. i}=\langle\phi(\Gamma) S(\Gamma)\rangle_{\Gamma}
$$

where $\langle\ldots \ldots .\rangle_{\Gamma}$ denotes an ensemble average over the distribution of static energies. If the dynamics and phase evolution are uncorrelated then it should be possible to factor Eq. (8) into the form

$$
S_{B}=\langle\phi(\Gamma)\rangle_{\Gamma}\langle S(k(\Gamma))\rangle_{\Gamma}
$$

Figure 5 shows model calculations for a two species inhomogeneous energy transfer ș'stem depicted in Figure 5a shows that while one-color 3PEPS (Fig. 6b) is unable to distinguish Eqs. (8) and (9). the 2C3PEPS experiment is very different for the two cases (Fig. 5c). The reason for the difference is that even though in the model system the donors and acceptors are initially energetically uncorrelated. the bias in the rate of energy transfer induced by the spectral overlap progressively correlates donors and acceptors. ${ }^{20}$

In the following two sections we describe two types of two-dimensional spectroscopy. In using the term two-dimensional. we follow the definition of $\mathrm{Cho}^{32}$ : an experiment is referred to as two-dimensional (2D) when two time delays or optical frequencies (or one of each) are independently controlled. We note that this definition excludes the type of experiment in which the second variable is a macroscopic one such as pressure. The important pressure-modulated infrared spectroscopy described by Noda ${ }^{43}$ with the goal of increasing spectral resolution by dispersing into a second dimension belongs in this latter category: A more restrictive definition of two-dimensional spectroscopy might be one in which two coherence periods are actively controlled via time delays (or frequency resolution). We will describe an experiment of this type - the fifth-order three-pulse scattering experiment (FOTS) or fifth-order three-pulse echo experiment (F3PE) in section III. but in general the two time periods may be either coherence or population periods in our definition of $2 \mathrm{D}$

We begin with a discussion of two-color photon echo peak shift spectroscopy (2C3PEPS).

Two-Color Three-Pulse Photon Echo Peak Shift (2C 3PEPS). The photon echo peak shift method has been described in detail in previous work..$^{9.11}$ It consists of measuring the delay from zero. $\tau^{*}(T)$, of the coherence period.

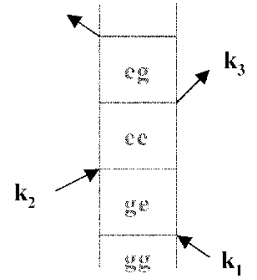

Type ] seat

Fcho) (Røphasing) (pulse sequence, $1-2-3$ )

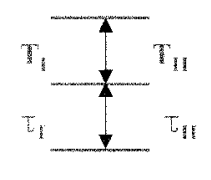

\section{$\mathbf{k}_{2}$}

Type II scout

FID (.רon-Rephasing!

(pulse sequente. 2-l-3) $\tau$. of the four-wave mixing signal as a function of the population period. $T$. Consider the two Feynman diagrams in Figure 6 . The peak slift goes to zero when there is no distinction between the left-hand (rephasing) diagram and the right hand (non-rephasing) diagram i.e. when the system has entirely lost memory of its transition frequency, $\omega_{\text {eg }}$. There are two important differences between one- and two-color peak shift experiments. First. one-color experiments. even those with very short pulses spanning the entire absorption band are dominated by degenerate four-wave mixing events. Thus. although the information present in two-color experiments must. in principle. be present in a one-color experiment carried out with identical pulses spanuing the same spectral range as the separate spectra of the two-color experiment. it can be completely buried under the degenerate contributions to the signal.

Second in the two-color case finite pulse-duration effects are of crucial importance, and the impulsive limit. so useful for developing simple models of photon echo experiments. is much less useful here. The difference between the two sequences shown in Figure 6. provided that pulse 3 is constrained to act last is simply the ordering of pulses 1 and 2. However. pulse ordering cannot be enforced when pulses 1 and 2 overlap in time making the interpretation of a 2C3PEPS plot of $\tau^{*}(T)$ «. T problematic. We have found that the influence of finite pulse duration can be greatly reduced by using two different scanning procedures to collect peak shift data - the conventional 3PEPS arrangement shown on the left of Figure 6 giving a quantity we define as $\tau_{1}^{*}\left(T_{j}\right)$. and a new arrangement based on the right side of Figure 6 giving $\tau_{I I}^{*}\left(T_{J}\right)$. Figure 6 shows that $\tau_{J}^{*}\left(T_{I I}\right)$ corresponds to a nonrephasing or free induction decay (FID) sequence. In this type of scan. the time between pulses 2 and 1 is called $\tau_{I I}$. with a positive value of $\tau_{I /}$ corresponding to pulse 2 interacting before pulse 1. The population period. $T_{I I}$. is the delay between pulses 1 and 3 . The most useful quantity is the difference peak shift $\Delta t^{*}(T)=\tau_{I}^{*}\left(T_{J}=T\right)-\tau_{I I}^{*}\left(T_{J}=T\right)$. When the system has no rephasing capability both types of scan will be identical and $\Delta \tau^{*}=0$. Otherwise we expect a finite value of $\Delta \tau^{*}$ and note that it may be a positive or a negative quantity depending on whether $\omega_{\mathrm{kg}}$ at the pump frequency is positively or negatively correlated with $\omega_{\mathrm{sg}}$ at the probe frequency:

Figure 7 shows model calculations for 2C3PEPS experiments on a dilute chromophore solution in which the first two interactions are at a higher frequency in the absorption band than the third interaction - we refer to this as a "downhill" 2C3PEPS experiment. The system is a polar chromophore interacting with a polar solvent and will therefore undergo typical solvation dỵnamics (Stokes shift) following excitation. Note that the type I and type II peak shifts start out at very similar values and then progressively diverge before becoming identical at about $500 \mathrm{fs}$. Thus the difference peak shift. $\Delta \tau^{*}(T)$. starts near zero. rises to a maximum and finally falls to zero by about $500 \mathrm{fs}$. This behavior is characteristic of that observed experimentally for
Figure 6. Example Feymant diagrams for the echo and FID pathway for the signal in the $\mathbf{k}_{3}+\mathbf{k}_{2}-\mathbf{k}_{1}$ phase matched direction. Note the reordering of the first 2 interactions and change in definition for the population time. similar sy stems as Figure 8 shows.

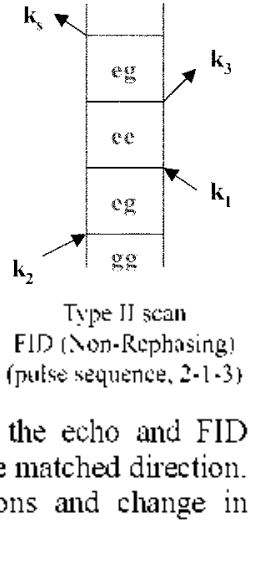



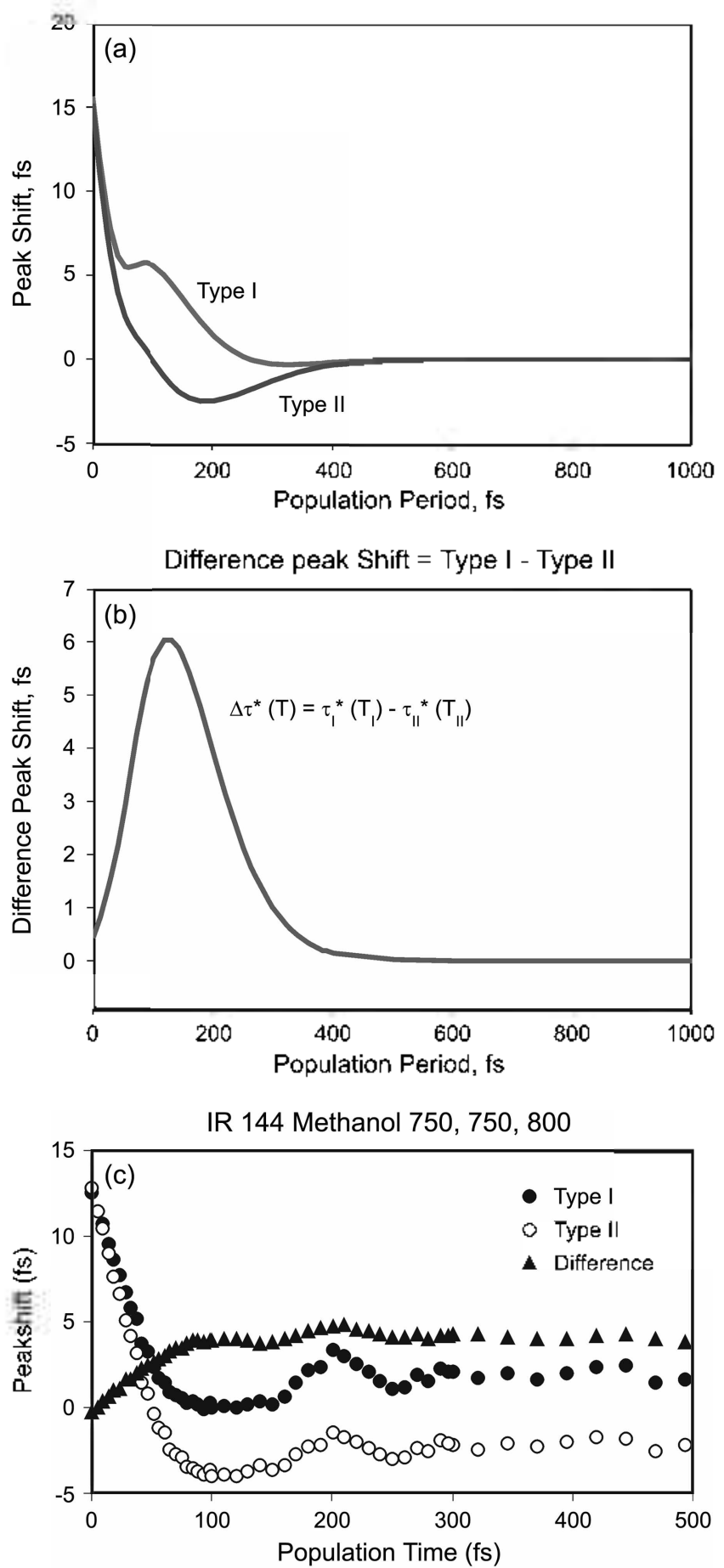

Figure 7. (a) Comparison of calculated type I and type II peak shifts for simple model system. (b) Calculated difterence peak shift for simple model system. (c) Ixperimental type [ (solid circles). type II (open circles). and difference peak shift (solid triangles) for IR I 44 in Methanol pumped with 2 pulses at 750 and probed with I at $800 \mathrm{~nm}$.

How should rigures 7 and 8 be interpreted? $\Lambda$ t the simplest level, the plot of $\Delta \tau^{*}$ rs. $T$ implies the rephasing capability is initially zero and increases to a maximum betore decaying. How can this be? In this system without energy transfer all the interactions of the laser pulses must be with the same molecule. Defining the tirst two interactions

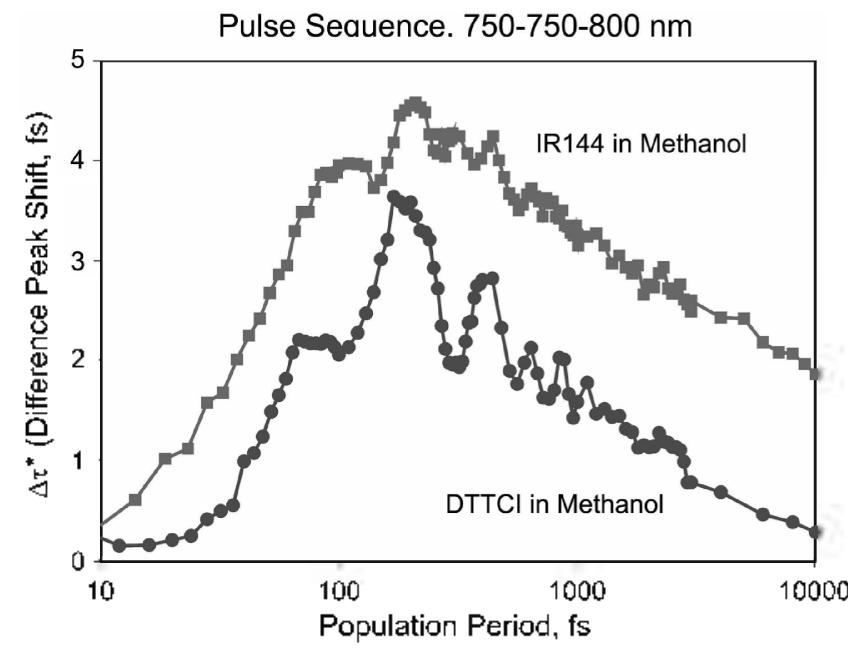

Figure 8. Fxperimental Difference Peak shifts tor IR 144 in Vethanol (solid squares) and I) I"I'Cl in Methanol (solid circles). The downhill pulse sequence of 750,750, $800 \mathrm{~nm}$ was used.

to be the pump. and the third to be the probe interaction, we consider two mechanisms contributing to the nonlinear signal. First, there can be overlap between the pump and probe spectral regions as a result of the homogeneous spectrum. I his distribution can be written

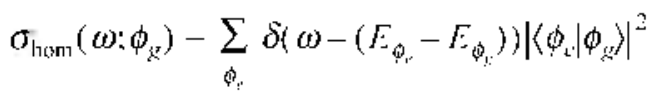

where $\phi_{g\left(x^{\prime}\right)}$ denotes a particular nuclear conliguration of the ground (excited) state. In other words. Fa. (10) just deseribes the vibronic structure of the transition.

The second contribution arises from evolution of the homogeneous distribution due to lluetuation and evolution (e.g. by the Stokes shift) of the inhomogeneous distribution. liere we simply write:

$$
\sigma(\omega)=\sum_{\phi_{i}} \sigma_{\mathrm{bom}}\left(\omega ; \phi_{k}\right) P_{\mathrm{kg}}\left(\phi_{\mathrm{k}}\right)
$$

where $P_{\mathrm{r}}$ is the stalistical probability for the molecule to oceupy the state $\phi_{g}$. Armed with these ideas we can construct a simple model to describe the time dependence of the response lunction $R\left(1: \omega_{p,}, \omega_{p t}\right)$ giving rise to the nonlinear signal:

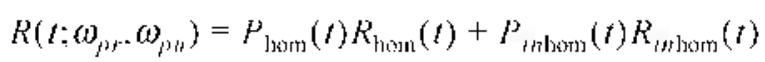

$\mathrm{Eq}+(12)$ is quite approximate - it is not possible to rigorously separate the response function in this mannerhowever it does give clcar physical insight. The physical behavior underlying $R\left(t ; \omega_{p, m} \omega_{p w}\right)$ is a comelation function deseribing the fluctuations of the transition energies in the pump and probe spectral regions. $\left\langle\delta \varepsilon_{p, r}(t) \delta \varepsilon_{p^{\prime}(t), p}(0)\right\rangle$. which we write as

$$
\begin{aligned}
& \left\langle\delta \varepsilon_{p, r}(t) \delta \varepsilon_{p t m p}(0)\right\rangle=\frac{P_{\text {homl }}(t)\left\langle\delta \varepsilon_{p \prime r}(t) \delta \varepsilon_{p t t}(0)\right\rangle_{\text {hom }}}{P_{\text {hom }}(t)+P_{\text {thlom }}(t)} \\
& +\frac{P_{\text {mhtimin }}(t)}{P_{\text {hom }}(t) \cdot P_{\text {inhom }}(t)}\left\langle\delta \varepsilon_{p r}(t) \delta \varepsilon_{p+t}(0)\right\rangle_{\text {inhum }}
\end{aligned}
$$




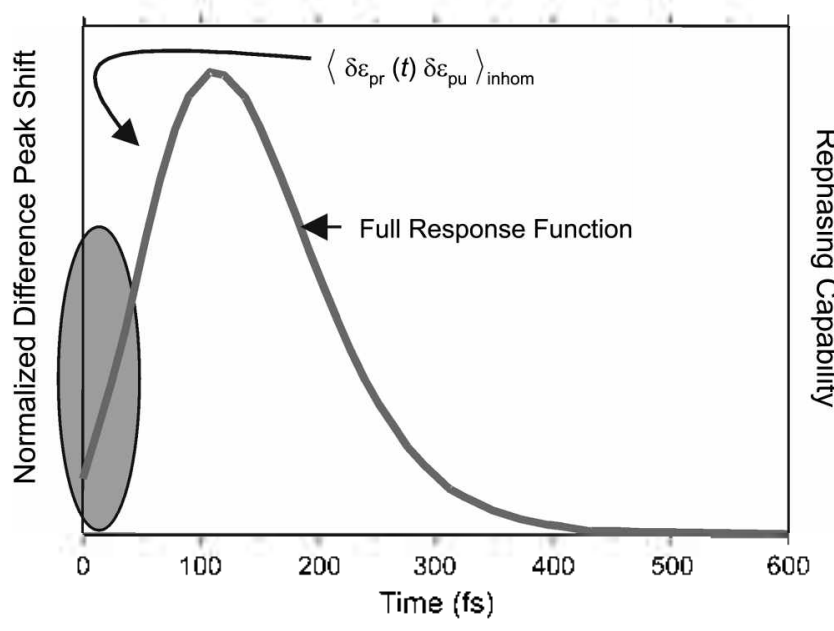

Figure 9. Comparison of the results of full response function numerical simulation of the ditterence peak shitt with a model for the rephasing capability given by the inhomogeneous part of equation 13 . The shaded oval denotes the time region where homogeneous broadening dominates the signal generation. See text.

At short times we expect $P_{\text {hom }}(t)>>P_{\text {inhom }}(t)$ while at longer times the influence of homogeneous broadening will be small: $P_{\text {hom }}(t) \ll<P_{\text {inthom }}(t)$. The dynamical behavior of $\Delta \tau(T)$, then is ultimately related to the evaluation of $\left\langle\delta \varepsilon_{y r}(t) \delta \varepsilon_{p r t}(0)\right\rangle_{\text {inhom }}$ which itself can be related to a time dependent conditional probability $P\left(\omega_{3} ; t \mid \omega_{1}\right)$ with $P\left(\omega_{2} ; 0 \mid \omega_{1}\right)=\delta\left(\omega_{2}-\omega_{1}\right)$. A number of authors have calculated forms for this conditional probability for systems undergoing solvation dynamics. ${ }^{44.47}$ The form derived by Skimer and coworkers ${ }^{47}$ clearly relates $P\left(\omega_{2} ; t \mid \omega_{1}\right)$ to $S(t)$ as defined in Eq. (4).

$$
\begin{aligned}
P\left(\omega_{2}: t \mid \omega_{1}\right) & =\frac{1}{\sqrt{2 \pi \Delta^{2}\left(1-S(t)^{2}\right)}} \\
& \times \exp \left\{\frac{\left.-\left[\left(\omega_{2}-\langle\omega\rangle\right)-(\omega)_{1}-\langle\omega\rangle\right) S(t)\right]^{2}}{2 \Delta^{2}\left(1-S(t)^{2}\right)}\right\}
\end{aligned}
$$

Figure 9 shows a plot of $\left\langle\delta \varepsilon_{p r}(t) \varepsilon_{p l}(0)\right\rangle$ obtained in this manner as compared with the response calculated from the full response function in which the homogeneous and inhomogeneous contributions are included (in a complicated way). The simple model clearly does poorly at short times where the homogeneous broadening is critical in signal formation. but does a good job in capturing the maximum in $\Delta \tau^{*}(T)$ and its subsequent decay. which is reproduced almost exactly. The only dynamical behavior included in the calculation of Figure 9 is the solvation dynamics i.e. the fluctuations and systematic red shift arising from the differential solvation energies of ground and excited states.

The rise in the difference peak shift in this picture should be connected with the inertial (menory conserving) component of the solvation dynamics. Simulations (Fig. 10) show this is so and further show a clear correlation in the rise of the downhill difference peak shift with the Gaussian time constant in model $S(t)$ functions. The subsequent decay of

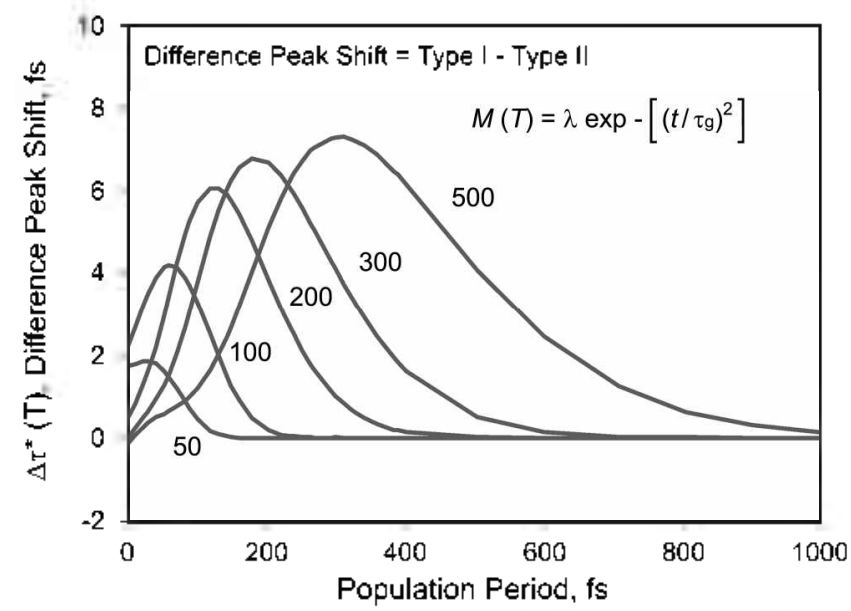

Figure 10. Model calculations for the difference peak shift for the downhill case. The $M /(t)$ consists of a single Gaussian mode with varying time constant.

$\Delta t^{*}(T)$ then corresponds to the diffusive component of the solvent response in which transition frequencies become scrambled in the ensemble.

In the companion paper in this volume on two-color transient grating spectroscopy. Cho and coworkers ${ }^{48}$ show that the initial portion of these signals also depends critically on the ultrafast initial component of the solvation correlation function.

Fifth Order Three-Pulse Scattering (FOTS). The fifth order three-pulse scattering experiment was proposed by Cho and Fleming in $1994^{22}$ as a way to separate homogeneous and inhomogeneous broadening in systems where the inhomogeneous width is comparable to the inverse of the timescale of the nuclear dy'namics. In such syistems. signals from conventional photon echo experiments contain both homogeneous and inhomogeneous contributions. The

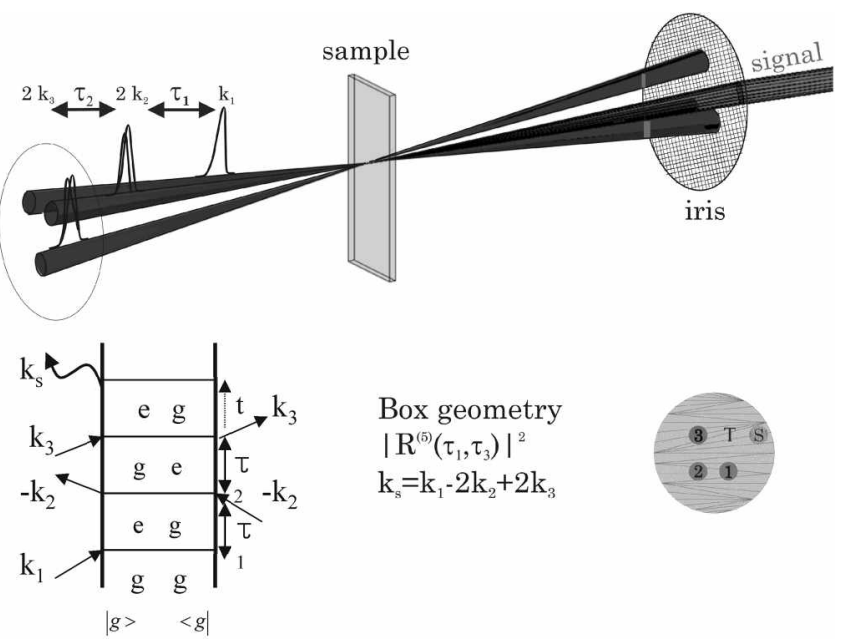

Figure 11. Fifth Order Resonant Experiment using 3 pulses. The st stem interacts once with the tirst pulse then twice with each of the remaining pulses as illustrated in the Fermmann diagram. The incoming pulses where ananged in a box gemetry with the signal detected in the $\mathbf{2} \mathrm{k}_{3}-\mathbf{2} \mathbf{k}_{2}+\mathrm{k}_{\text {l }}$ phase matched direction. 
experiment was first carried out by Joo et al. (in this work the technique is referred to as F3PE). ${ }^{+5}$ Figure 11 shows a Feymman diagram pulse sequence and optical layout for FOTS. In an FOTS experiment the system is placed in a electronic coherence by the first pulse and evolves for a time period $\tau_{1}$. The second pulse involves two interactions which leave the system in an electronic coherence which is the complex conjugate of the first. During $\tau_{\hat{3}}$. the system rephases. The third pulse also interacts twice leaving the syrstem in a coherence which is the same as that following the first pulse. Thus the electronic polarization will fully rephase when $\tau_{3}=\tau_{1}+t$ provided the phase memory is not lost. In FOTS. both $\tau_{1}$ and $\tau_{2}$ are controllable. allowing control over the rephasing process that is not possible in conventional echo measurements. In the work of Joo et al ${ }^{45}$ only slices at particular fixed values of $\tau_{1}$, along $\tau_{2}$ were recorded along with the diagonal slice along $\tau_{1}=\tau_{2}$. In this paper we present the first full two-dimensional surfaces for
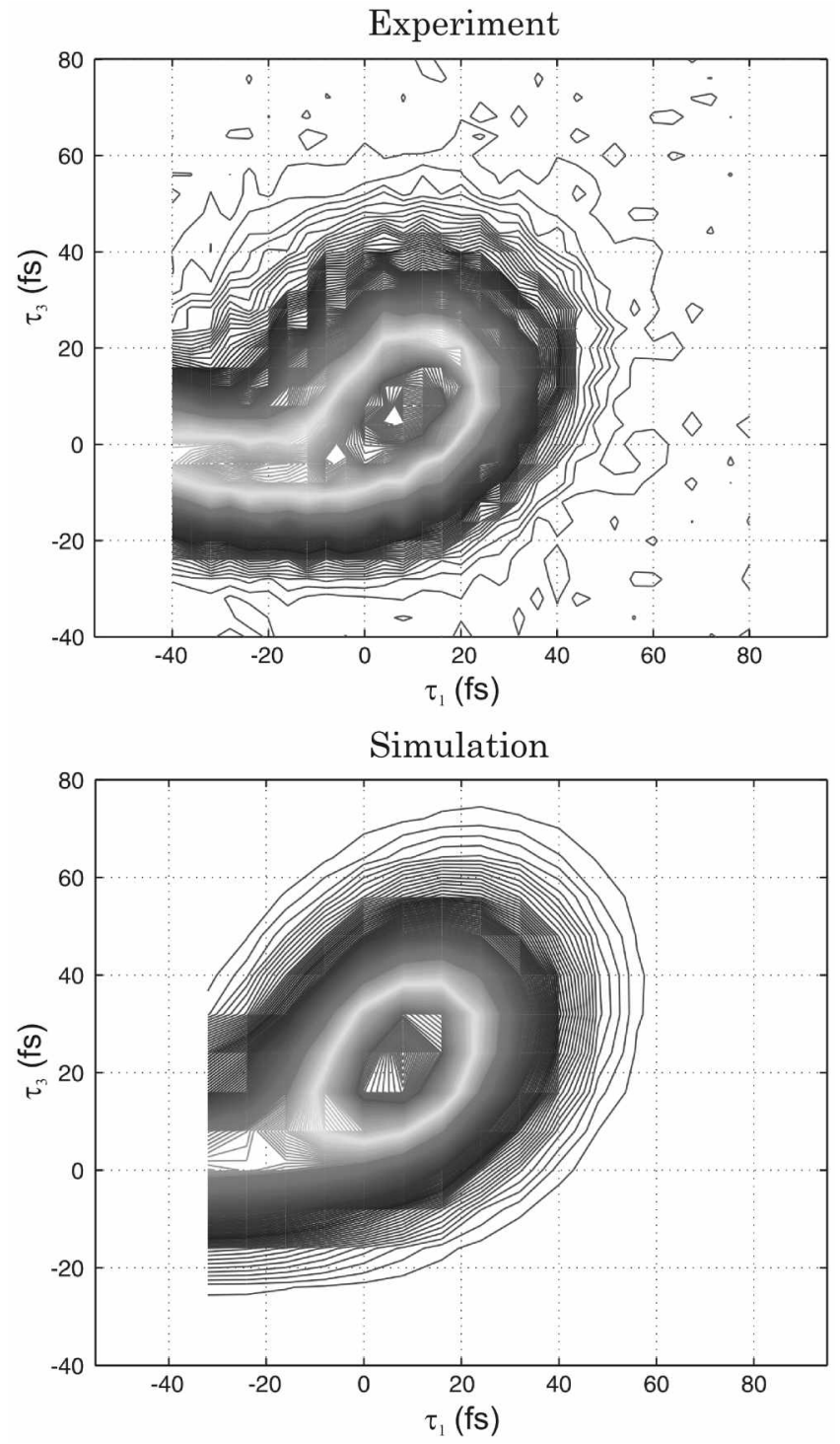

Figure 12. Fifth Order Three Pulse Scattering of IR 144 in methanol as a function the two coherence periods. Excellent agreement is seen between experiment and simulation. the FOTS signal as a function of $\tau_{1}$ and $\tau_{2}$

Figure 12 shows the two-dimensional FOTS surface for a dilute solution of the dy'e molecule IR144 in methanol. The upper panel shows the experimental data and the lower a calculated surface based on a solvation model for IR $144^{9}$ Several aspects of the data are immediately apparent: Because the experiment contains no population period and three coherence periods. the signal is expected to decay very quickly. Indeed the signal is very small by $60 \mathrm{fs}$ in both $\tau_{1}$ and th dimensions. Second over the whole duration for which the surface could be recorded the intensity is maximum along the diagonal $\tau_{1}=\tau_{\hat{\Omega}}$ line. No sign of a turnover toward a constant value of $\tau_{\hat{\Omega}}$ for increasing $\tau_{1}$ is seen. clearly showing that the system is exhibiting echo belavior for times significantly beyond the $60 \mathrm{fs}$ that is observed. Thus the Gaussian (time reversible) nature of the initial component of the solvation correlation function. $S(t)$ is confirmed. In the most commonly used third-order echo experiment - the three-pulse photon echo peak shift method (3PEPS) - the initial decay of the signal does not correspond to $S(t)$ directly ${ }^{1 !}$ and it has been difficult to confinm that the initial decay is indeed Gaussian as expected.

The question arises as to whether there is more infomation present in principle beyond better characterization of the two-time correlation function $S(t)$ or $M(t)$ in the FOTS experiment than in a third order experiment such as 3PEPS. In general, the higher the order of the experiment, the higher the order of the correlation function that can be measured. Thus 3PEPS can be described with only two-time correlation functions while FOTS, in principle can provide threetime correlation function information and hence more detailed knowledge of the underlying system dynamics. However, in some cases the higher order experiment will not provide more infonmation than the third order experiment because the sy stem dynamics is completely characterized by a two-time correlation function. For example. if the model of coupling of a two electronic-level system to a hanmonic bath is a good model for the șistem dỵamics. then all experiments of no matter what order can be described by a twotime correlation function. For simple two-level syystems coupled to a harmonic bath, the FOTS experiment may lead to a better characterization of the two-time correlation function, but is unlikely to lead to qualitatively new information

New types of information, not available at third order. can be revealed by fifth-order experiments on șystems exhibiting anharmonicity of some kind. For example. excitonically coupled sy stems give rise to electronic states that do not look like hamonic energy levels. Thus dynamical phenomena associated with intemolecular coupling should be better understood by comparing the results of third and fifth order experiments for example the electron-phonon coupling may vary from one exciton level to another because of the varying degrees of charge transfer etc. Knowing how to extract this information is quite another story! A second example of an anhanmonic electronic signal which exists in the absence of intermolecular coupling is the electronic 


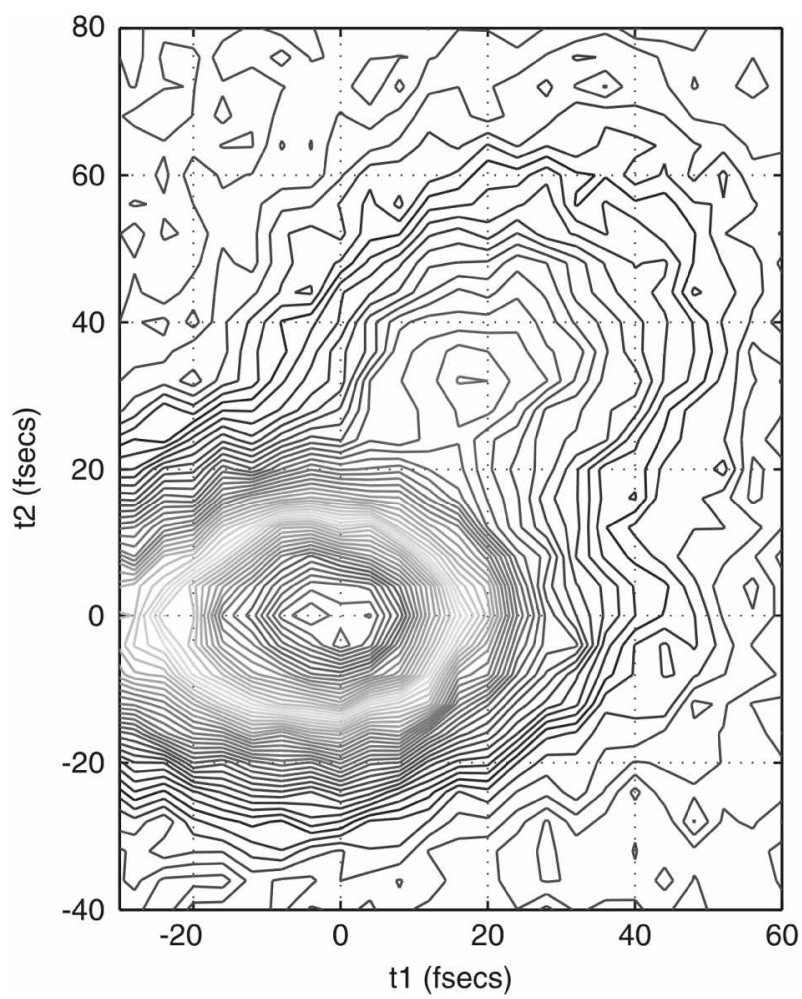

Figure 13. Fifth Order Three Pulse Scattering of LH2 B800-B850 complex of Rps acidophila obtained with 17 fs excitation pulses capable of exciting both the $\mathrm{B} 800$ and $\mathrm{B} 850$ bands.

structure of a molecular complex (in the exciton picture) with correlated disorder. Thus. the nature of disorder in such systems may be significantly clarified by higher order spectroscopy.

Such measurements. let alone their analysis. are in their infancy. Figure 13 shows the FOTS signal for the LH2 B800-B850 complex of Rps acidophila ${ }^{513}$ excited at $800 \mathrm{~nm}$ with $17 \mathrm{fs}$ pulses. The bandwidth of the pulses is sufficient to excite both the 18 excitonically coupled B 820 molecules and the 9 more weakly coupled B800 molecules. The overall structure of the surface differs significantly from the dilute dye solution case. The strong peak centered at $\tau_{1}=\tau_{2}=0$ is not tilted along the diagonal and the diagonal signal is much weaker and does not appear until $\tau_{1}$ and $\tau_{2}$ are $>20 \mathrm{fs}$. The strong feature around $\tau_{1}=\tau_{2}=0$ may be the result of scattering because the signal from the nuclear dynamics is much weaker than the IR $144 / \mathrm{MeOH}$ system. The diagonal structure however. clearly originates from electron/nuclear dynamics and appears to persist until at least 60fs. Despite the relatively poor signal to noise ratio. the surface appears to tilt towards the $\tau_{2}$ axis for $\tau_{2}>50 \mathrm{fs}$. It seems likely that the tilt towards $\tau_{3}$ arises from ultrafast dephasing from the exciton relaxation among the B820 molecules. however no detailed analysis of the surface in Figure 13 has yet been performed. With the recent advances in our understanding of the electronic structure quantum dynamics and nature of disorder in such systems ${ }^{51.15 .52 .53}$ such a calculation should be possible in the near future.

\section{Concluding Comments}

Two-dimensional electronic spectroscopy is an emerging new form of optical spectroscopy. It can be implemented in a substantial number of ways ranging from the elegant approach of Jonas and coworkers ${ }^{54}$ through the two-color experiments described here and in ref 26. the fifth order experiment described above. and the variety of two-dimensional correlation spectroscopies described by Mukamel. ${ }^{42}$ As the implementation of heterodyne detection ${ }^{55-59}$ becomes increasingly common. the various forms of two-dimensional spectroscopy will exhibit their full infomation content and be applied to the unraveling of dynamical properties of a wide range of complex systems whose dynamical behavior cannot be described by a two-level system coupled to a harmonic bath.

Acknowledgement. This work was supported by a grant from the NSF.

\section{References}

1. Meech. S. R.: Hoof. A. J.; Weirsna, D. A. Chen. Phws Lett 1985. 121. 287.

2. Weiner. A. M.: De Silvestri. S.: Ippen. E. P. J. Opt. Soc. Am. B 1985. 2.654.

3. Becker. P. C.: Fragnito. H. L.: Bigot. T. Y.: Brito Cruz. C. H.: Fork. R. L.; Shank, C. V. Phus Rev: Lett. 1989. 63. 505.

4. Bigot. J.Y.; Portella, M. I; Schoenlein. R. W. Bardeen. C. J: Migus. A.: Shank, C. V. Phys. Rev Lett. 1991. 66. 1138.

5. Nibbering. E. T. J.: Wiersma. D. A.: Duppen. K. Phns. Rev Lett. 1991.66 .2464$.

6. Vohringer. P.: Arnett. D. C.: Westervelt. R. A.: Feldstein. M. T.: Scherer. N. F. J. Chem Phws. 1995. 102. 4027.

7. Joo. T: Albrecht, A. C. Chent. Phns. 1993. 176. 233

8. Joo. T: Jia, Y; Fleming, G. R. J. Chem. Plys. 1995, 102.4063.

9. Joo. T.: Tia. Y.: Yu. J.Y.: Lang. M. T.: Fleming. G. R. J. Chem Phys. 1996. 104. 6089.

10. Cho. M.: Yu. T.-Y.: Joo. T.: Nagasawa. Y.: Passino. S. A.: Fleming. G. R. J. Phos Chem. 1996. 100.11944.

11. de Boeij. W. P.; Pshenichnikov, M. S.; Wiersma. D. A. Chent. Phys. Lett. 1995. 238. 1

12. Agarwal. R.: Krueger. B.: Scholes. G. D.: Yang. M.: Yom. J.: Mets. L.: Fleming. G. R. J. Phys. Chem. B 2000. 104. 2908.

13. Agarwal. R.: Yang. M.: Xu. Q.-H.: Fleming. G. R. J. Phus Chent $B$ 2001, 105. 1887 .

14. Yu, J.-Y.: Nagasawa, Y; van Grondelle, R.: Fleming, G. R. Chent Phys. Lett. 1997. 280. 404.

15. Agarwal. R.: Rizvi. A. H.: Prall. B. S.: Olsen. J. D.: Hunter. C. N.: Fleming. G. R. J. Plys. Chem A 2002. 106. 7573.

16. Jimenez. R.: yan Mourik. F.: Yu. J. Y.: Fleming. G. R. J. Phrs. Chen. B 1997. 101.7350.

17. Groot. M.-L.; Yu. J.-Y.: Agarwal. R; Noris, J. R.; Fleming. G. R. $J P C B 1998,102.5923$.

18. Ohta. K.: Yang. M.: Fleming. G. R. J. Chem. Plns. 2001. 115. 76019.

19. Yantg. M.: Fleming. G. R. J. Chem. Phys. 1999. 111.27.

20. Yang. M.: Fleming. G. R. J. Chent. Plins. 2000. 113. 2823.

21. Hybl. J. D.: Ferro, A. A.: Jonas, D. M. J. Chem. Phos. 2001.115. 6606.

22. Cho. M.: Fleming, G. R. J. Phus. Chem. 1994, 98.3478.

23. Mukamel. S. Am Rev Phys. Chem 2000.51.691

24. Faeder. S. M. G.: Jonas. D. J. Phys. Chem A 1999. 1-3. 10489.

25. Yantg. M.: Fleming. G. R. J. Chent. Phws. 1999. 110. 2983.

26. Agarwal, R.; Prall. B. S.; Rizvi. A. H; Yang. M.: Fleming, G. R. $J$ 
Chem. Phws. 2002. 116.6243.

27. Zwanzig. R. J. Stat. Plys. 1973. 9. 215.

28. Mukamel. S. Principles of Nonhinear Optical Spectroscopy: Onford University Press: New York. 1995.

29. Fleming. G. R.: Cho. M. Annt. Rev Plns Chem. 1996. 47. 109

30. Jordanides. X. J.: Lang. M. J.: Song. X.: Fleming. G. R. J. Plns. Chem. B 1999, 103, 7995.

31. Lang, M. I.: Iordanides. X. J:- Song. X.: Fleming, G. R. J. Chem. Phys. 1999. 110.5884.

32. Cho. M. H. Phs them. Comm 2002. 7. 40.

33. Cho. M. H. J. Chem. Phns 2001. MH. 8040

34. Underwood. D. F: Blank. D. A. J. Phys. Chem. A 2003, 107, 956.

35. Jordan. P: Fromme. P.: Witt, H. T: Klukas, O.: Saenger. W.: Krauss. N. Hature 2001. H1. 909.

36. Chitnis. P. R. Amm. Rev: Plam Plysiol. Plam Mol. Biol. 2001. 52. 593.

37. McDermott G.: Prince. S. M: Freer. A. A: HawthonthwaiteLawless. A. M.: Papiz, M. Z.: Cogdell. R. J.: Isaacs. N. W. Kature $1995,374,517$

38. De Caro. C.: Visschers. R. W.: van Grondelle. R.: Völker. S. J. The Journal of Physical Chemistry 1994.98. 10584.

39. Hess. S.: Feldchtein. F.: Rabin. A.: Nurgaleev. I.: Pullerits. T. Sergeet. A.: Sundströn. V. Chemical Phnsics Letters 1993, 216. 247.

40. Jimenez, R.: Dikshit S. N.: Bradforth. S. E.: Fleming, G. R. J. Plys. Chem. B 1996. 100.6825.

41. Raetsep. M.: Johnson. T. W.: Chitnis. P. R.: Small. G. J. J. Phns. Chem. B 2000. 104. 836 .

42. Mukamel S. Am Rev Phis Chem. 2000. 51.691.
43. Noda I.: Story. G. M.; Marcott. C. Iibrational Spectroscopy 1999. 19. 461.

44. Kinoshita. S. 7. Chem. Phus. 1989. 91.5175.

45. Loring. R. F.: Yan. Y. T.: Mukamel. S. J. Chem. Plys. 1987. 87. 5840 .

46. Mukamel. S. J. Chem. Phws 1983. 79. 2126.

47. Stephens, M. D.; Saven. J. G; Skinner. J. L. J. Chent. Phos 1997. 106. 2129

48. Kwak. K. W.: Cho. M. H.: Fleming. G. R.: Agarwal. R.: Prall. B S. Bull. Korean Chem. Soc. 2003. 24. In press.

49. Joo. T.: Jia. Y.: Fleming. G. R. J. Chent. Phns. 1995. 102.4063.

50. Neuwahl, F.; Kaufman. L. J.: Agarwal, R: Fleming, G. R umpublished results.

51. Kennis. J. T. M; Streltsov, A. M: Permentier, H; Aartsma, T. J;; Amesz. J. The Jounal of Physical Chemisty B 1997. 101. 8369.

52. Yang. M.: Agarwal. R.: Fleming. G. R. J. Photochem Photobio. At 2001. $1+2.107$.

53. Yang. M.; Fleming, G. R. Chem. Phws. 2002. 275, 355.

54. Hybl. J. D.; Faeder. S. M. G.: Albrecht, A. W. Tolbert, C. A.; Green. D. C.: Jonas. D. M. J. Lamin. 2000. 126.87.

55. de Boeij. W. P.: Pshenichnikov. M. S.: Wiersma. D. A. Chem. Phys. Lett. 1995. 247.264.

56. Goodno. G. D.: Dadues. G.: Miller. R. J. D. J. Opt. Soc. Ant B 1998. $15,1791$.

57. Khalil. M; Demirdoevon, Ni; Golonzka. O.: Fecko, C. J.: Tokmakof, A. J. Plys. Chem \& 2000. 104, 5711 .

58. Kaufmant. L. J.: Heo. J. Y.: Zeigler. L. D.: Fleming. G. R. Phys. Rev Lett. 2002. 88.207.

59. Xu. Q. H.: Ma. Y. Z.: Fleming. G. R. Chent. Phns. Lett. 2001.338. 254. 